\title{
Quantitative proteomics to study carbapenem resistance in Acinetobacter baumannii
}

\author{
Vishvanath Tiwari * and Monalisa Tiwari \\ Department of Biochemistry, Central University of Rajasthan, Ajmer, India
}

\section{Edited by:}

Ana Cristina Esteves, University of Aveiro, Portugal

Reviewed by:

Benjamin Andrew Evans, Anglia

Ruskin University, UK

Bart Devreese, Ghent University,

Belgium

\section{*Correspondence:}

Vishvanath Tiwari, Department of

Biochemistry, Central University of

Rajasthan, Ajmer,

Rajasthan 305817, India

e-mail: vishvanath7@yahoo.co.in
Acinetobacter baumannii is an opportunistic pathogen causing pneumonia, respiratory infections and urinary tract infections. The prevalence of this lethal pathogen increases gradually in the clinical setup where it can grow on artificial surfaces, utilize ethanol as a carbon source. Moreover it resists desiccation. Carbapenems, a $\beta$-lactam, are the most commonly prescribed drugs against $A$. baumannii. Resistance against carbapenem has emerged in Acinetobacter baumannii which can create significant health problems and is responsible for high morbidity and mortality. With the development of quantitative proteomics, a considerable progress has been made in the study of carbapenem resistance of Acinetobacter baumannii. Recent updates showed that quantitative proteomics has now emerged as an important tool to understand the carbapenem resistance mechanism in Acinetobacter baumannii. Present review also highlights the complementary nature of different quantitative proteomic methods used to study carbapenem resistance and suggests to combine multiple proteomic methods for understanding the response to antibiotics by Acinetobacter baumannii.

Keywords: quantitative proteomics, Acinetobacter baumannii, carbapenem resistance, pathogenesis, biofilm formation

\section{INTRODUCTION}

Acinetobacter baumannii is one of the six opportunistic pathogens grouped into ESKAPE pathogens that are linked to the highest degree of mortality as described by Infectious Disease Society of America (Klevens et al., 2006; Boucher et al., 2009). It causes pneumonia, urinary tract infections, respiratory infections and meningitis. Acinetobacter baumannii has emerged as a threat to soldiers, wounded during military operations in Iraq and Afghanistan (Davis et al., 2005). The organism can be isolated from natural resources (Kempf et al., 2012). It can grow on artificial surfaces (Espinal et al., 2012) and can utilize ethanol as a carbon source (Navon-Venezia et al., 2005; Fiester and Actis, 2013; Gandhi et al., 2014), resist desiccation, survive at various temperature and $\mathrm{pH}$ conditions (Bergogne-Berezin and Towner, 1996), this makes it a lethal pathogen. Prevalence of Acinetobacter baumannii in clinical setup has increased gradually (Tiwari et al., 2012a). Commonly prescribed drug against A. baumannii are carbapenems which belong to the $\beta$-lactam group of antibiotics (Hawkey and Jones, 2009). Resistance against carbapenem has emerged in Acinetobacter baumannii which can create significant health problems and responsible for high morbidity and mortality (Sengstock et al., 2010). Reports showed that mortality due to carbapenem-resistant Acinetobacter baumannii is about $52 \%$ as compared to $19 \%$ when infected with carbapenem sensitive variant (Jamulitrat et al., 2009). This makes it one of the major health concerns.

Quantitative proteomics has been employed for both discovery and targeted proteomic analysis to understand global proteomic dynamics of the organism. With the development of quantitative proteomics, a considerable progress has been made in the study of drug resistance. There are various methods which are used in the quantitative proteomics. Oldest method is the comparison of two commassie stained 2D-PAGE gels (Klose, 1975; O'farrell, 1975; Meleady, 2011; Rabilloud and Lelong, 2011). This was followed by emergence of labeled method like Differential In-Gel Electrophoresis, DIGE (Unlu et al., 1997; Yan et al., 2002; Knowles et al., 2003; Timms and Cramer, 2008), Stable Isotope Labeling by Amino acids in Cell culture, SILAC (Ong et al., 2002; Ong and Mann, 2005) Isotope Coded Affinity Tags, ICAT (Gygi et al., 1999; Gygi and Aebersold, 2000), Isobaric tags quantification, iTRAQ (Ross et al., 2004; Desouza et al., 2009) and Isotope-coded protein label, ICPL (Kellermann, 2008; Abdallah et al., 2012; Kellermann and Lottspeich, 2012). Isotope labels can be introduced into peptides metabolically, chemically or enzymatically. Most recently developed methods for quantitative proteomics are label free quantification methods like spectral counting (Mirza and Olivier, 2008; Zhu et al., 2010) and Selected Reaction Monitoring, SRM (Elschenbroich and Kislinger, 2011; Hossain et al., 2011). In label free proteomic methods, mass spectrometer can recognize the mass difference and their quantification is achieved by comparing their respective signal intensities. Proteomic techniques have been used for the identification and quantification of protein samples and its validation have been done by ELISA (Chen et al., 2010), western blotting (Xiaoyu et al., 2013), immunohistochemistry (Perdomo et al., 2012), RT-PCR and real time PCR (Choi and Shim, 2008; Zhang et al., 2011; Paul et al., 2013; Xiaoyu et al., 2013). In the present review, we critically review the use of quantitative proteomics in the study of carbapenem resistance in Acinetobacter baumannii. We also discuss the future perspective of the quantitative proteomics in the study of carbapenem resistance 
as well as limitation of different methods used in quantitative proteomics.

\section{QUANTITATIVE PROTEOMICS AS A TOOL TO STUDY OF CARBAPENEM RESISTANCE IN A. BAUMANNII}

Quantitative proteomics has very diverse applications and significance but in the present review, we have explained its significance in the study of carbapenem resistance of Acinetobacter baumannii. A number of approaches using quantitative proteomics have been employed for the study of bacterial drug resistance and pathogenesis.

Quantitative outer membrane proteomics between wild type and carbapenem resistance strain of Acinetobacter baumannii have been reported (Marti et al., 2006; Siroy et al., 2006; Kwon et al., 2009). Using silver stained 2DE gel, selection of protein spots based on the intensity and sharpness of the spots in the gel; and identification of the protein by homologous matching with other species of Acinetobacter, Marti et al., performed an analysis of the major proteins in the membrane fraction of a multidrug resistant strain of Acinetobacter baumannii and identify OmpA, ribosomal protein, chaperone and elongation factor (Marti et al., 2006). The result does not highlight the quantitative expression of protein but it signifies the application of the proteomics in the study of carbapenem resistance. Siroy et. al., performed global comparison of the membrane fraction of a sensitive strain with a carbapenem resistant strain of Acinetobacter baumannii by comparing two coomassie stained 2D-PAGE gels. Results highlighted that about 36 and $56 \%$ protein spots were different in the inner membrane and outer membrane fraction of resistant strain of Acinetobacter respectively as compared to the sensitive strain (Siroy et al., 2006). They pointed out that resistance against carbapenem has been developed due to overexpression of RND-type efflux systems and virulence factors like FepA-like and siderophore receptors, absence of PBP1b protein, structural modifications to the CarO porin and presence of different isoforms of the channel OmpW in the carbapenem resistant strain of Acinetobacter baumannii (Siroy et al., 2006). This study has given a new direction to the study of carbapenem resistance using quantitative proteomics approach. Similar to approach of Marti et al. (2006), Kwon et al., also used silver stained 2DE gel to identify 132 proteins associated with outer membrane vesicles of Acinetobacter baumannii (Kwon et al., 2009). 2D electrophoresis was also used to explain role of different proteins in metabolism using native ATCC strain of Acinetobacter baumannii (Soares et al., 2009b). Results highlighted that robust and versatile metabolism of Acinetobacter plays very important role in the carbapenem resistance and virulence of $A$. baumannii (Soares et al., 2009b). Using 2DE based membrane proteomic approach; Lee et al., explained the mechanism of hetero-resistance induced by imipenem (a member of carbapenem group) in the multidrug resistant Acinetobacter baumannii (Lee et al., 2011). They showed that imipenem treatment leads to the up-regulation of AmpC, Cpn60 chaperonin, ATP synthase, and OmpA (Lee et al., 2011).

Vashist et al., showed the importance of outer membrane in the carbapenem resistance using differential DIGE of outer membrane of carbapenem resistance strain as compared to sensitive strain of Acinetobacter (Vashist et al., 2010). They concluded that emergence of carbapenem resistance in A. baumannii is due to the decreased expression of CarO, porins (e.g., Omp-A) and increased expression of biofilm forming protein (e.g., CsuA/B) and nutrient transporters (e.g., iron binding protein, $\mathrm{ABC}$ transporter). Vashist et al., identified more proteins with significant role in the carbapenem resistance of Acinetobacter as compare to Siroy et al. (2006), which further confirm the advantages of DIGE based methods (Vashist et al., 2010) over silver stained methods (Siroy et al., 2006). Similarly, Tiwari et al., identified the importance of the metabolism in the carbapenem resistance of Acinetobacter using differential DIGE of inner membrane fraction (Tiwari et al., 2012c). Results concluded that emergence of carbapenem resistance in A. baumannii was found to be associated with overproduction of carbapenem hydrolysing $\beta$ lactamase (e.g., AmpC and OXA-51) and metabolic enzymes as well as downregulation of surface antigen and OmpW. They also showed that overproduction of these proteins/enzymes have been achieved by enhanced transcription, translation (e.g., Elongation factor $\mathrm{Tu}$ and $30 \mathrm{~S}$ ribosomal protein) and folding (e.g., $60 \mathrm{KDa}$ chaperonin and TCP-1/cpn60 chaperonin protein (Tiwari et al., 2012c).

Yun et al., performed differential quantitative proteomic analysis of cell wall and plasma membrane fractions from multidrug-resistant Acinetobacter baumannii using labeled iTRAQ quantitative approach (Yun et al., 2011). They reported that carbapenem also induces the expression of resistancenodulation-cell division transporters, protein kinases and suppresses outer membrane proteins expression (Yun et al., 2011). This study further advanced the study of carbapenem resistance of Acinetobacter baumannii because it used isobaric tag for labeling, reverse phase chromatography for peptide separation and MS/MS mass spectrometry for peptide identification which was relatively more sensitive than older methods. They also compared the result from label as well as label free methods and concluded that more than $80 \%$ protein have similar expression (down/up-regulation) pattern for label-free (LC-MS/MS) and labeled (iTRAQ) methods (Yun et al., 2011). This result highlighted the importance to use more than one quantitative proteomic method because of their complementary nature to enhance the reproducibility and validity of quantitative proteomic result. The identified proteins from different quantitative proteomic methods have been listed in the Table 1.

Pathogenesis of the Acinetobacter is also influenced by hostpathogen interaction and nutritional immunity of the host. Role of nutritional immunity in the survival of carbapenem resistance strain of Acinetobacter in human host has also been studied using differential proteomic approach (Nwugo et al., 2011; Mortensen and Skaar, 2012; Tiwari, 2013). Nwugo et al., compare the 2DE gel of total lysate and outer membrane fractions isolated from A. baumannii sensitive strain cultured under iron-rich and irondeficient condition. Results indicated that iron-rich condition leads to the overexpression of proteins involved in the iron storage, metabolic process and lipid biosynthesis while iron deficient condition leads to the overexpression of proteins involved in the iron acquisition (Nwugo et al., 2011). Similarly, using DIGE and LC-MS/MS, Tiwari et al., concluded that carbapenem resistant strain of A. baumannii upregulates proteins associated with 
Table 1 | Differentially expressed proteins identified in the carbapenem resistance strain as compared to sensitive strain of $A$. baumannii using various quantitative proteomic approaches (Siroy et al., 2006; Kwon et al., 2009; Vashist et al., 2010; Cabral et al., 2011; Lee et al., 2011; McQueary and Actis, 2011; Yun et al., 2011; Tiwari et al., 2012c).

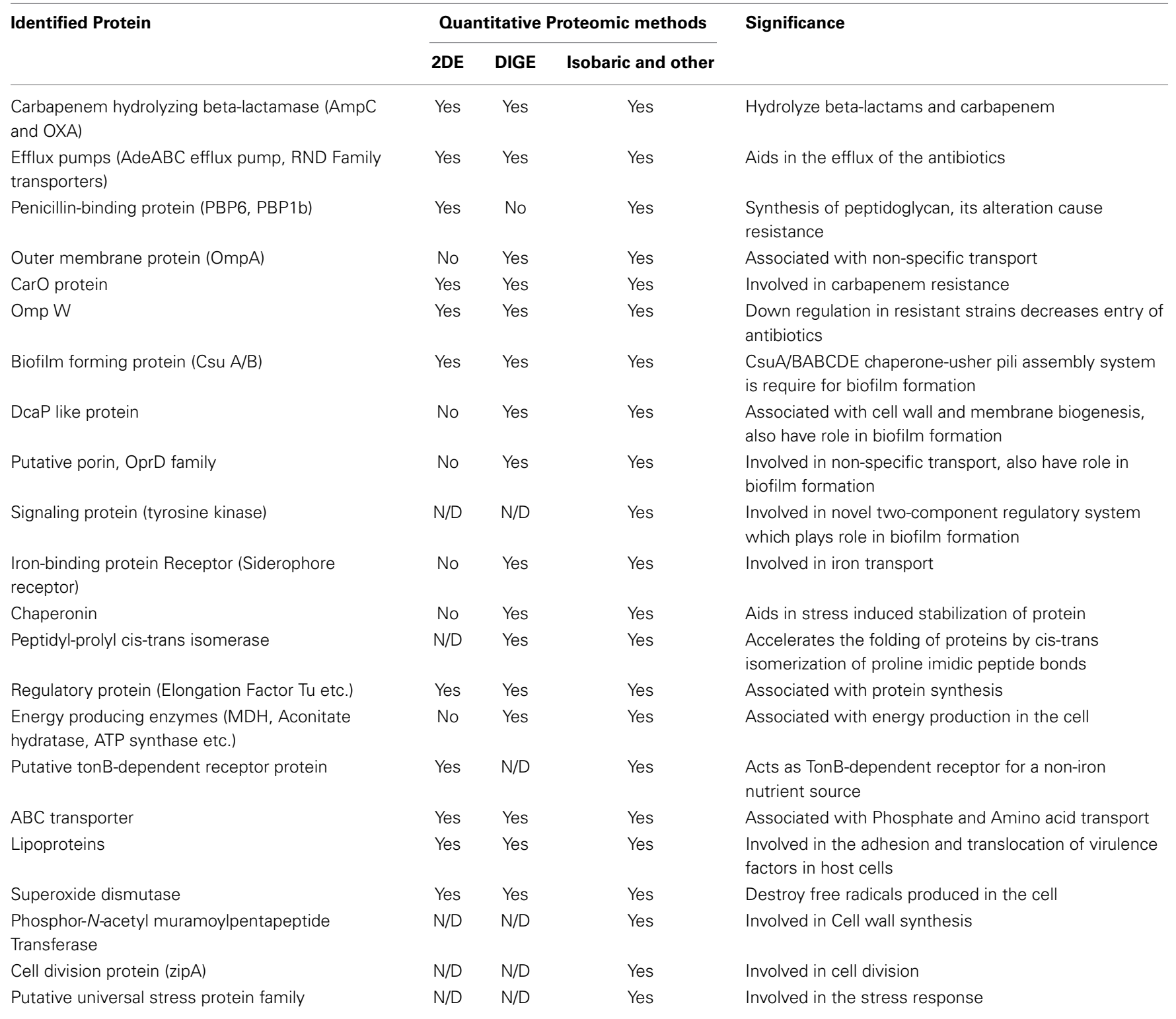

No, No change; Yes, changed in the resistant strain as compared to sensitive strain; N/D, Not detected.

iron acquisition under iron limiting condition while upregulate metabolic enzymes under iron limiting condition (Tiwari, 2013).

High-end isoelectric point ( $\mathrm{pH}$ 6-11) differential proteome analysis of Acinetobacter radioresistens also reveals that envelope stress responses can be induced by aromatic compounds (Mazzoli et al., 2011). Role of proteins detected by the quantitative proteomics methods has been validated by other in-vitro or in-vivo methods. It was found that Acinetobacter baumannii develop resistance against carbapenem via alteration in the expression/activity of $\beta$-lactamase (Tiwari et al., 2012a,b,c; Tiwari, 2013, 2014; Tiwari and Moganty, 2013, 2014) and alteration in the penicillin binding protein (Vashist et al., 2011). Upregulation of metabolic enzymes/proteins found in the inner membrane protein also enhanced the carbapenem resistance in A. baumannii (Tiwari et al., 2012c).

Biofilm is a functional consortium of microorganisms organized within an extensive exopolymeric matrix (Gurung et al., 2013). Biofilm formation is one of the important causes for the persistence of Acinetobacter baumannii on the surface of host epithelial cells and other surfaces (Espinal et al., 2012; Longo et al., 2014). Cabral et al., performed differential proteomics of Acinetobacter cultured in three different conditions (exponential, late stationary phase and biofilms stage) using 2D-DIGE and MALDI-TOF/TOF as well as iTRAQ/SCX-LC-MS/MS. They also checked the effects of biofilm inhibitory compound (salicylate) on the biofilm formation. This multiple-approach strategy showed 
a unique lifestyle of $A$. baumannii involved in biofilm formation (Cabral et al., 2011). CsuA/BABCDE chaperone-usher pili assembly system have been identified in Acinetobacter as essential for biofilm formation on plastic (McQueary and Actis, 2011). OmpA and CarO have role in the uptake of amino acid hence also have role in biofilm formation by Acinetobacter baumannii (Cabral et al., 2011).

Acinetobacter baumannii OMV's induces pathogenesis in the host because of secretion of the outer membrane protein (Kwon et al., 2009) which is cytotoxic to the host (Jin et al., 2011). Secretion of OMVs from A. baumannii has been studied using silver stained 2DE based quantitative proteomics approach (Kwon et al., 2009). Soares et al., identified alterations in the plasma proteome of individuals infected with Acinetobacter baumannii as compared to healthy controls using DIGE based differential proteomic approach (Soares et al., 2009a).

Using quantitative phosphoproteomics approach, selected phosphorylation sites have been identified in Acinetobacter which has been discussed in the context of stress/starvation, pathogenicity and drug resistance (Soares et al., 2014). Qualitative comparison between the Ser/Thr/Tyr phosphoproteomes employed SCX and $\mathrm{TiO}_{2}$ chromatography for enrichment of phosphopeptide and LTQ-Orbitrap mass spectrometric analysis for phosphopeptide identification. The percentage distribution of Ser/Thr/Tyr phosphorylation was found to be $68.9 \%$ for serine, $24.1 \%$ for threonine and $5.2 \%$ for tyrosine in sensitive strain as compared to $70.8 \%$ for serine, $25.2 \%$ for threonine and $3.8 \%$ for tyrosine in carbapenem resistant strain of A. baumannii (Soares et al., 2014). Phosphoproteomics identified 70 phosphoproteins in the multidrug resistant strain (AbH12O-A2) of A. baumannii as compared to 41 phosphoproteins in the sensitive strain (ATCC 17978) of Acinetobacter (Soares et al., 2014). Identified phosphoproteins play role in the pathogenesis (e.g., PtK), virulence (e.g., $\mathrm{KdpD} / \mathrm{KdpE}$ ) and drug resistance (Soares et al., 2014). PtK significantly enhances the ability of Acinetobacter to grow in human biofluids. $\mathrm{KdpD} / \mathrm{KdpE}$, a bacterial two-component signal transduction system, have role in the virulence-related regulatory functions (Soares et al., 2014). This study highlight the significance of high throughput quantitative methods for the study of signaling associated with carbapenem resistance strain of Acinetobacter baumannii.

Induction of proteins associated with signaling, putative virulence factors and various stress responses at different stages of invitro growth has been identified using growth phase-dependent quantitative proteomics using 2-DE and MALDI-TOF/TOF complemented by iTRAQ and LC-MS/MS (Soares et al., 2010; Fiester and Actis, 2013). This result further highlighted the significance of multiple quantitative approaches used together. Extracellular proteome of Acinetobacter baumannii has been characterized using 2DE and nanoLC-MS/MS based quantitative proteomic approach (Mendez et al., 2012). Mendez et al., used two protein fractions of the extracellular proteome i.e., outer membrane vesicle $(\mathrm{OMV})$ proteins and freely soluble extracellular proteins (FSEPs) present in the culture medium of A. baumannii. The result showed that OMV proteins were found to be associated with pathogenesis and virulence (e.g., CsuE, CsuB, CsuA/B) and secretion systems for delivery of virulence factors while FSEP fraction have extracellular enzymes with degradative activity and role in oxidative stress response (Mendez et al., 2012).

Proteomic experiments also identified differentially expressed lipoproteins as well as proteins responsible for inflammatory/coagulation pathways and kallikrein-kinin system of Acinetobacter which will improve future developments in the pathogenesis of the Acinetobacter and its therapy (Soares et al., 2009a). In quantitative immunoproteomic approach, potentially immunogenic proteins in A. baumannii have been identified using 2DE and MALDI-TOF/TOF mass spectrometric analysis. Immunogenic proteins could serve as antigen for the development of vaccines and passive immunotherapies against A. baumannii infections (Bonin et al., 2014).

\section{LIMITATION OF DIFFERENT QUANTITATIVE PROTEOMIC METHODS AND THEIR SIGNIFICANCE IN THE CARBAPENEM RESISTANCE STUDIES}

There are number of limitations of quantitative proteomic methods that hamper the study of the carbapenem resistance mechanism of Acinetobacter baumannii. Merits and demerit of different methods used in the quantitative proteomics have been listed in the Table 2.

Table 2 showed that most of the methods used in quantitative proteomics did not provide information about posttranslational modification and they are unable to detect the small proteins. These two limitations can cost heavily in the studies of carbapenem resistance. Quantitative proteomics is unable to produce all information required to confirm role of protein/biomolecule in carbapenem resistance but it gives global insights about resistance mechanism of Acinetobacter baumannii which helps in the subsequent studies. Quantitative proteomic methods are also unable to give direct confirmation of the role of protein in the carbapenem resistance. Similarly the result of quantitative proteomics is also influenced by protein extraction procedure. These limitations conclude that quantitative proteomics help to a large extent in the study of carbapenem resistance but it requires help of other methods for confirmation. Therefore, we can say that emergence of quantitative proteomics is really an added advantage in the study of carbapenem resistance.

\section{FUTURE PERSPECTIVES OF QUANTITATIVE PROTEOMICS TO UNDERSTAND CARBAPENEM RESISTANCE}

Fluorescence based DIGE methods and isobaric based iTRAQ methods in combination with LC-MS/MS are more popular approach used to study carbapenem resistance mechanism of A. baumannii. Phosphoproteomics and immunoproteomics have also emerged to understand virulence and pathogenesis of carbapenem resistant strain of $A$. baumannii. Every proteomic method has its own merits and demerits (Table 2) but they provide complementary information. Therefore, combining multiple methods together is an added advantage. This approach has been recently used by some groups to produce valid results (Cabral et al., 2011; Yun et al., 2011). Literature based on proteomic methods has showed the presence of differentially expressed proteins in the carbapenem resistant strain but their actual role in carbapenem resistance has not been confirmed 
Table 2 | Different quantitative proteomic approaches with its merits and demerits.

\begin{tabular}{llll}
\hline Proteomic Tools & Merits & Demerits & References \\
\hline GEL-BASED METHODS & & Klose, 1975; O'farrell, 1975; \\
$2 D E$ & (1) Simplistic & (1) Involve large amount of sample & Meleady, 2011; Rabilloud and \\
& (2) Robust & (2) Low throughput & Lelong, 2011
\end{tabular}

$\begin{array}{ll}\text { 2D-DIGE } & \text { (1) Multiplexing } \\ \text { (2) Better quantitation } \\ \text { (3) Minimized gel to gel variation }\end{array}$

(1) Expensive Cy dyes

(2) Poor recovery of hydrophobic proteins

(3) Difficulty in separation of low molecular weight
Unlu et al., 1997; Yan et al., 2002; Knowles et al., 2003; Timms and Cramer, 2008; Chen et al., 2010

GEL-FREE METHODS

\begin{tabular}{|c|c|c|c|}
\hline SILAC & $\begin{array}{l}\text { (1) High throughput } \\
\text { (2) Robust } \\
\text { (3) Sensitive and simple }\end{array}$ & $\begin{array}{l}\text { (1) Suitable only for tissue culture models } \\
\text { (2) Costly reagents } \\
\text { (3) Not applicable to tissue sample }\end{array}$ & $\begin{array}{l}\text { Ong et al., 2002; Ong and Mann, } \\
\text { 2005; Elliott et al., } 2009\end{array}$ \\
\hline ICAT & $\begin{array}{l}\text { (1) Selectively isolates peptide } \\
\text { (2) Compatible with any amount } \\
\text { of protein } \\
\text { (3) Complexity of the peptide } \\
\text { mixture is reduced }\end{array}$ & $\begin{array}{l}\text { (1) Cannot identify proteins with less than } \\
8 \text { cysteines } \\
\text { (2) Size of ICAT label is large }(\approx 500 D a) \\
\text { (3) Post-translational modification } \\
\text { information is frequently lost }\end{array}$ & $\begin{array}{l}\text { Gygi et al., 1999; Gygi and } \\
\text { Aebersold, 2000; Toyo'oka, } 2012\end{array}$ \\
\hline iTRAO & $\begin{array}{l}\text { (1) Applicable to versatile samples } \\
\text { (2) Multiplexing } \\
\text { (3) Better quantitation }\end{array}$ & $\begin{array}{l}\text { (1) Involve high amount of sample } \\
\text { (2) Incomplete labeling } \\
\text { (3) Expensive reagents }\end{array}$ & $\begin{array}{l}\text { Ross et al., 2004; Desouza et al., } \\
2009\end{array}$ \\
\hline ICPL & $\begin{array}{l}\text { (1) High-throughput quantitative } \\
\text { proteome profiling on a global } \\
\text { scale } \\
\text { (2) Able to detect } \\
\text { post-translational modifications } \\
\text { and protein isoforms } \\
\text { (3) Applicable to protein sample } \\
\text { like extracts from tissues or body } \\
\text { fluids }\end{array}$ & $\begin{array}{l}\text { (1) Isotopic effect of deuterated tags that } \\
\text { interferes with retention time of the } \\
\text { labeled peptides during LC }\end{array}$ & $\begin{array}{l}\text { Kellermann, 2008; Abdallah et al., } \\
2012 \text {; Kellermann and Lottspeich, } \\
2012\end{array}$ \\
\hline Label-free & $\begin{array}{l}\text { (1) Involve less amount of sample } \\
\text { (2) Higher proteome coverage } \\
\text { (3) Avoid labeling }\end{array}$ & $\begin{array}{l}\text { (1) High throughput instrumentation } \\
\text { (2) Not suitable for low abundant proteins } \\
\text { (3) Incomplete digestion may introduce } \\
\text { error } \\
\text { (4) Multiplexed analysis is not possible in } \\
\text { one experiment }\end{array}$ & $\begin{array}{l}\text { Mirza and Olivier, 2008; Zhu } \\
\text { et al., } 2010\end{array}$ \\
\hline SRM & $\begin{array}{l}\text { (1) Highly sensitive, quantitatively } \\
\text { accurate and highly reproducible } \\
\text { (2) Protein detection is relatively } \\
\text { rapid and straightforward } \\
\text { (3) Enable the detection of low } \\
\text { (>10 ng/ml) abundance proteins } \\
\text { (4) Quantification of } \\
\text { post-translational modification }\end{array}$ & $\begin{array}{l}\text { (1) Limited broad scale application } \\
\text { because of difficulty of generating } \\
\text { high-quality SRM assay. } \\
\text { (2) Sensitivity is not comparable to } \\
\text { immunological assays } \\
\text { (3) Detection and quantification of low } \\
\text { abundance proteins (i.e., } \sim 10 \mathrm{ng} / \mathrm{ml} \text { or } \\
\text { less) }\end{array}$ & $\begin{array}{l}\text { Elschenbroich and Kislinger, 2011; } \\
\text { Hossain et al., } 2011\end{array}$ \\
\hline
\end{tabular}

using knockout or auxotroph for resistance factors. Therefore, quantitative proteomic methods also require support from nonproteomic methods. With the development of the quantitative immunoproteomics, phosphoproteomics and other omics methods, the identification of the differentially expressed resistance factors become more reliable. Emergence of quantitative proteomic methods like ICPL and SRM methods will help to remove the shortcoming of the routinely used present methods. Future of quantitative proteomics will depend on the use of two quantitative proteomic methods together with better validation methods to outcome the limitation of the current methods or approach. 


\section{CONCLUSIONS}

The quantitative proteomics has overcome some of the limitations of other approaches for investigating carbapenem resistance. With the development in the quantitative proteomics, new opportunities are now open to study difficult and challenging tasks. Because of complementary nature of different quantitative proteomic methods, it has been suggested to combine multiple quantitative proteomic methods for the better understanding of carbapenem resistance in Acinetobacter baumannii.

\section{ACKNOWLEDGMENT}

Vishvanath Tiwari would like to thanks UGC, India and SERB, DST, India for providing Start-up Grant.

\section{REFERENCES}

Abdallah, C., Dumas-Gaudot, E., Renaut, J., and Sergeant, K. (2012). Gel-based and gel-free quantitative proteomics approaches at a glance. Int. J. Plant Genomics 2012:494572. doi: 10.1155/2012/494572

Bergogne-Berezin, E., and Towner, K. J. (1996). Acinetobacter spp. as nosocomial pathogens: microbiological, clinical, and epidemiological features. Clin. Microbiol. Rev. 9, 148-165.

Bonin, R. F., Chapeaurouge, A., Perales, J., Da Silva Junior, J. G., Do Nascimento, H. J., D'alincourt Carvalho Assef, A. P., et al. (2014). Identification of immunogenic proteins of the bacterium Acinetobacter baumannii using a proteomic approach. Proteomics Clin. Appl. doi: 10.1002/prca.201300133. [Epub ahead of print].

Boucher, H. W., Talbot, G. H., Bradley, J. S., Edwards, J. E., Gilbert, D., Rice, L. B., et al. (2009). Bad bugs, no drugs: no ESKAPE! An update from the infectious diseases society of America. Clin. Infect. Dis. 48, 1-12. doi: 10.1086/ 595011

Cabral, M. P., Soares, N. C., Aranda, J., Parreira, J. R., Rumbo, C., Poza, M., et al. (2011). Proteomic and functional analyses reveal a unique lifestyle for Acinetobacter baumannii biofilms and a key role for histidine metabolism. J. Proteome Res. 10, 3399-3417. doi: 10.1021/pr101299j

Chen, Y. T., Chen, C. L., Chen, H. W., Chung, T., Wu, C. C., Chen, C. D., et al. (2010). Discovery of novel bladder cancer biomarkers by comparative urine proteomics using iTRAQ technology. J. Proteome Res. 9, 5803-5815. doi: $10.1021 / \mathrm{pr} 100576 \mathrm{x}$

Choi, Y. E., and Shim, W. B. (2008). Identification of genes associated with fumonisin biosynthesis in Fusarium verticillioides via proteomics and quantitative real-time PCR. J. Microbiol. Biotechnol. 18, 648-657.

Davis, K. A., Moran, K. A., McAllister, C. K., and Gray, P. J. (2005). Multidrugresistant Acinetobacter extremity infections in soldiers. Emerging Infect. Dis. 11, 1218-1224. doi: 10.3201/eid1108.050103

Desouza, L. V., Romaschin, A. D., Colgan, T. J., and Siu, K. W. (2009). Absolute quantification of potential cancer markers in clinical tissue homogenates using multiple reaction monitoring on a hybrid triple quadrupole/linear ion trap tandem mass spectrometer. Anal. Chem. 81, 3462-3470. doi: 10.1021/ac802726a

Elliott, M. H., Smith, D. S., Parker, C. E., and Borchers, C. (2009). Current trends in quantitative proteomics. J. Mass Spectrom. 44, 1637-1660. doi: 10.1002/jms.1692

Elschenbroich, S., and Kislinger, T. (2011). Targeted proteomics by selected reaction monitoring mass spectrometry: applications to systems biology and biomarker discovery. Mol. Biosyst. 7, 292-303. doi: 10.1039/c0mb00159g

Espinal, P., Marti, S., and Vila, J. (2012). Effect of biofilm formation on the survival of Acinetobacter baumannii on dry surfaces. J. Hosp. Infect. 80, 56-60. doi: 10.1016/j.jhin.2011.08.013

Fiester, S. E., and Actis, L. A. (2013). Stress responses in the opportunistic pathogen Acinetobacter baumannii. Future Microbiol. 8, 353-365. doi: 10.2217/fmb.12.150

Gandhi, J. A., Ekhar, V. V., Asplund, M. B., Abdulkareem, A. F., Ahmadi, M., Coelho, C., et al. (2014). Alcohol enhances Acinetobacter baumannii-associated pneumonia and systemic dissemination by impairing neutrophil antimicrobial activity in a murine model of infection. PLOS ONE 9:e95707. doi: 10.1371/journal.pone.0095707

Gurung, J., Khyriem, A. B., Banik, A., Lyngdoh, W. V., Choudhury, B., and Bhattacharyya, P. (2013). Association of biofilm production with multidrug resistance among clinical isolates of Acinetobacter baumannii and Pseudomonas aeruginosa from intensive care unit. Indian J. Crit. Care Med. 17, 214-218. doi: 10.4103/0972-5229.118416

Gygi, S. P., and Aebersold, R. (2000). Mass spectrometry and proteomics. Curr. Opin. Chem. Biol. 4, 489-494. doi: 10.1016/S1367-5931(00)00121-6

Gygi, S. P., Rist, B., Gerber, S. A., Turecek, F., Gelb, M. H., and Aebersold, R. (1999). Quantitative analysis of complex protein mixtures using isotope-coded affinity tags. Nat. Biotechnol. 17, 994-999. doi: 10.1038/13690

Hawkey, P. M., and Jones, A. M. (2009). The changing epidemiology of resistance. J. Antimicrob. Chemother. 64 Suppl. 1, i3-10. doi: 10.1093/jac/dkp256

Hossain, M., Kaleta, D. T., Robinson, E. W., Liu, T., Zhao, R., Page, J. S., et al. (2011). Enhanced sensitivity for selected reaction monitoring mass spectrometry-based targeted proteomics using a dual stage electrodynamic ion funnel interface. Mol. Cell Proteomics 10, M000062-MCP000201. doi: 10.1074/mcp.M000062MCP201

Jamulitrat, S., Arunpan, P., and Phainuphong, P. (2009). Attributable mortality of imipenem-resistant nosocomial Acinetobacter baumannii bloodstream infection. J. Med. Assoc. Thai. 92, 413-419.

Jin, J. S., Kwon, S. O., Moon, D. C., Gurung, M., Lee, J. H., Kim, S. I., et al. (2011). Acinetobacter baumannii secretes cytotoxic outer membrane protein A via outer membrane vesicles. PLoS ONE 6:e17027. doi: 10.1371/journal.pone.0017027

Kellermann, J. (2008). ICPL—isotope-coded protein label. Methods Mol. Biol. 424, 113-123. doi: 10.1007/978-1-60327-064-9_10

Kellermann, J., and Lottspeich, F. (2012). Isotope-coded protein label. Methods Mol. Biol. 893, 143-153. doi: 10.1007/978-1-61779-885-6_11

Kempf, M., Bakour, S., Flaudrops, C., Berrazeg, M., Brunel, J. M., Drissi, M., et al. (2012). Rapid detection of carbapenem resistance in Acinetobacter baumannii using matrix-assisted laser desorption ionization-time of flight mass spectrometry. PLoS ONE 7:e31676. doi: 10.1371/journal.pone.0031676

Klevens, R. M., Edwards, J. R., Tenover, F. C., McDonald, L. C., Horan, T., Gaynes, R., et al. (2006). Changes in the epidemiology of methicillin-resistant Staphylococcus aureus in intensive care units in US hospitals, 1992-2003. Clin. Infect. Dis. 42, 389-391. doi: 10.1086/499367

Klose, J. (1975). Protein mapping by combined isoelectric focusing and electrophoresis of mouse tissues. A novel approach to testing for induced point mutations in mammals. Humangenetik 26, 231-243.

Knowles, M. R., Cervino, S., Skynner, H. A., Hunt, S. P., De Felipe, C., Salim, K., et al. (2003). Multiplex proteomic analysis by two-dimensional differential ingel electrophoresis. Proteomics 3, 1162-1171. doi: 10.1002/pmic.200300437

Kwon, S. O., Gho, Y. S., Lee, J. C., and Kim, S. I. (2009). Proteome analysis of outer membrane vesicles from a clinical Acinetobacter baumannii isolate. FEMS Microbiol. Lett. 297, 150-156. doi: 10.1111/j.1574-6968.2009.01669.x

Lee, H. Y., Chen, C. L., Wang, S. B., Su, L. H., Chen, S. H., Liu, S. Y., et al. (2011). Imipenem heteroresistance induced by imipenem in multidrugresistant Acinetobacter baumannii: mechanism and clinical implications. Int. J. Antimicrob. Agents 37, 302-308. doi: 10.1016/j.ijantimicag.2010.12.015

Longo, F., Vuotto, C., and Donelli, G. (2014). Biofilm formation in Acinetobacter baumannii. New Microbiol. 37, 119-127.

Marti, S., Sanchez-Cespedes, J., Oliveira, E., Bellido, D., Giralt, E., and Vila, J. (2006). Proteomic analysis of a fraction enriched in cell envelope proteins of Acinetobacter baumannii. Proteomics 6 Suppl. 1, S82-S87. doi: 10.1002/pmic.200500323

Mazzoli, R., Fattori, P., Lamberti, C., Giuffrida, M. G., Zapponi, M., Giunta, C., et al. (2011). High isoelectric point sub-proteome analysis of Acinetobacter radioresistens $\mathrm{S} 13$ reveals envelope stress responses induced by aromatic compounds. Mol. Biosyst. 7, 598-607. doi: 10.1039/c0mb00112k

McQueary, C. N., and Actis, L. A. (2011). Acinetobacter baumannii biofilms: variations among strains and correlations with other cell properties. J. Microbiol. 49, 243-250. doi: 10.1007/s12275-011-0343-7

Meleady, P. (2011). 2D gel electrophoresis and mass spectrometry identification and analysis of proteins. Methods Mol. Biol. 784, 123-137. doi: 10.1007/978-161779-289-2_9

Mendez, J. A., Soares, N. C., Mateos, J., Gayoso, C., Rumbo, C., Aranda, J., et al. (2012). Extracellular proteome of a highly invasive multidrug-resistant clinical strain of Acinetobacter baumannii. J. Proteome Res. 11, 5678-5694. doi: $10.1021 /$ pr300496c

Mirza, S. P., and Olivier, M. (2008). Methods and approaches for the comprehensive characterization and quantification of cellular proteomes using mass spectrometry. Physiol. Genomics 33, 3-11. doi: 10.1152/physiolgenomics. 00292.2007 
Mortensen, B. L., and Skaar, E. P. (2012). Host-microbe interactions that shape the pathogenesis of Acinetobacter baumannii infection. Cell. Microbiol. 14, 1336-1344. doi: 10.1111/j.1462-5822.2012.01817.x

Navon-Venezia, S., Ben-Ami, R., and Carmeli, Y. (2005). Update on Pseudomonas aeruginosa and Acinetobacter baumannii infections in the healthcare setting. Curr. Opin. Infect. Dis. 18, 306-313. doi: 10.1097/01.qco.0000171920.44809.f0

Nwugo, C. C., Gaddy, J. A., Zimbler, D. L., and Actis, L. A. (2011). Deciphering the iron response in Acinetobacter baumannii: a proteomics approach. J. Proteomics 74, 44-58. doi: 10.1016/j.jprot.2010.07.010

O'farrell, P. H. (1975). High resolution two-dimensional electrophoresis of proteins. J. Biol. Chem. 250, 4007-4021.

Ong, S. E., Blagoev, B., Kratchmarova, I., Kristensen, D. B., Steen, H., Pandey, A., et al. (2002). Stable isotope labeling by amino acids in cell culture, SILAC, as a simple and accurate approach to expression proteomics. Mol. Cell. Proteomics 1, 376-386. doi: 10.1074/mcp.M200025-MCP200

Ong, S. E., and Mann, M. (2005). Mass spectrometry-based proteomics turns quantitative. Nat. Chem. Biol. 1, 252-262. doi: 10.1038/nchembio736

Paul, D., Kumar, A., Gajbhiye, A., Santra, M. K., and Srikanth, R. (2013). Mass spectrometry-based proteomics in molecular diagnostics: discovery of cancer biomarkers using tissue culture. Biomed Res. Int. 2013, 783131. doi: $10.1155 / 2013 / 783131$

Perdomo, A. B., Ciccosanti, F., Iacono, O. L., Angeletti, C., Corazzari, M., Daniele, N., et al. (2012). Liver protein profiling in chronic hepatitis C: identification of potential predictive markers for interferon therapy outcome. J. Proteome Res. 11, 717-727. doi: 10.1021/pr2006445

Rabilloud, T., and Lelong, C. (2011). Two-dimensional gel electrophoresis in proteomics: a tutorial. J. Proteomics 74, 1829-1841. doi: 10.1016/j.jprot.2011.05.040

Ross, P. L., Huang, Y. N., Marchese, J. N., Williamson, B., Parker, K., Hattan, S., et al. (2004). Multiplexed protein quantitation in Saccharomyces cerevisiae using amine-reactive isobaric tagging reagents. Mol. Cell. Proteomics 3, 1154-1169. doi: 10.1074/mcp.M400129-MCP200

Sengstock, D. M., Thyagarajan, R., Apalara, J., Mira, A., Chopra, T., and Kaye, K. S. (2010). Multidrug-resistant Acinetobacter baumannii: an emerging pathogen among older adults in community hospitals and nursing homes. Clin. Infect. Dis. 50, 1611-1616. doi: 10.1086/652759

Siroy, A., Cosette, P., Seyer, D., Lemaitre-Guillier, C., Vallenet, D., Van Dorsselaer, A., et al. (2006). Global comparison of the membrane subproteomes between a multidrug-resistant Acinetobacter baumannii strain and a reference strain. J. Proteome Res. 5, 3385-3398. doi: 10.1021/pr060372s

Soares, A. J., Santos, M. F., Trugilho, M. R., Neves-Ferreira, A. G., Perales, J., and Domont, G. B. (2009a). Differential proteomics of the plasma of individuals with sepsis caused by Acinetobacter baumannii. J. Proteomics 73, 267-278. doi: 10.1016/j.jprot.2009.09.010

Soares, N. C., Cabral, M. P., Gayoso, C., Mallo, S., Rodriguez-Velo, P., FernandezMoreira, E., et al. (2010). Associating growth-phase-related changes in the proteome of Acinetobacter baumannii with increased resistance to oxidative stress. J. Proteome Res. 9, 1951-1964. doi: 10.1021/pr901116r

Soares, N. C., Cabral, M. P., Parreira, J. R., Gayoso, C., Barba, M. J., and Bou, G. (2009b). 2-DE analysis indicates that Acinetobacter baumannii displays a robust and versatile metabolism. Proteome Sci. 7, 37. doi: 10.1186/1477-5956-7-37

Soares, N. C., Spat, P., Mendez, J. A., Nakedi, K., Aranda, J., and Bou, G. (2014). Ser/Thr/Tyr phosphoproteome characterization of Acinetobacter baumannii: comparison between a reference strain and a highly invasive multidrug-resistant clinical isolate. J. Proteomics 102, 113-124. doi: 10.1016/j.jprot.2014.03.009

Timms, J. F., and Cramer, R. (2008). Difference gel electrophoresis. Proteomics 8, 4886-4897. doi: 10.1002/pmic. 200800298

Tiwari, V. (2013). Effect of iron availability on the survival of carbapenem-resistant Acinetobacter baumannii: a proteomic approach. J. Proteomics Bioinform. 6, 125-131. doi: 10.4172/jpb.1000270

Tiwari, V. (2014). Membrane Proteomics has emerged as a tool to study carbapenem resistance in Acinetobacter baumannii. J. Proteomics Bioinform. 7:e26 doi: $10.4172 /$ jpb.10000e26

Tiwari, V., Kapil, A., and Moganty, R. R. (2012a). Carbapenem-hydrolyzing oxacillinase in high resistant strains of Acinetobacter baumannii isolated from India. Microb. Pathog. 53, 81-86. doi: 10.1016/j.micpath.2012.05.004
Tiwari, V., and Moganty, R. R. (2013). Structural studies on New Delhi Metallo$\beta$-lactamase (NDM-2) suggest old $\beta$-lactam, penicillin to be better antibiotic for NDM-2-harbouring Acinetobacter baumanni. J. Biomol. Struct. Dyn. 31, 591-601. doi: 10.1080/07391102.2012.706075

Tiwari, V., and Moganty, R. R. (2014). Conformational stability of OXA-51 $\beta$ lactamase explains its role in carbapenem resistance of Acinetobacter baumannii. J. Biomol. Struct. Dyn. 32, 1406-1420. doi: 10.1080/07391102.2013.819789

Tiwari, V., Nagpal, I., Subbarao, N., and Moganty, R. R. (2012b). In-silico modeling of a novel OXA-51 from beta-lactam-resistant Acinetobacter baumannii and its interaction with various antibiotics. J. Mol. Model. 18, 3351-3361. doi: 10.1007/s00894-011-1346-3

Tiwari, V., Vashistt, J., Kapil, A., and Moganty, R. R. (2012c). Comparative proteomics of inner membrane fraction from carbapenem-resistant Acinetobacter baumannii with a reference strain. PLoS ONE 7:e39451. doi: 10.1371/journal.pone.0039451

Toyo'oka, T. (2012). LC-MS determination of bioactive molecules based upon stable isotope-coded derivatization method. J. Pharm. Biomed. Anal. 69, 174-184. doi: 10.1016/j.jpba.2012.04.018

Unlu, M., Morgan, M. E., and Minden, J. S. (1997). Difference gel electrophoresis: a single gel method for detecting changes in protein extracts. Electrophoresis 18, 2071-2077. doi: 10.1002/elps.1150181133

Vashist, J., Tiwari, V., Das, R., Kapil, A., and Rajeswari, M. R. (2011). Analysis of penicillin-binding proteins (PBPs) in carbapenem resistant Acinetobacter baumannii. Indian J. Med. Res. 133, 332-338.

Vashist, J., Tiwari, V., Kapil, A., and Rajeswari, M. R. (2010). Quantitative profiling and identification of outer membrane proteins of beta-lactam resistant strain of Acinetobacter baumannii. J. Proteome Res. 9, 1121-1128. doi: 10.1021/ pr9011188

Xiaoyu, L., Weiyuan, Z., Ping, J., Anxia, W., and Liane, Z. (2013). Comparative serum proteomic analysis of adenomyosis using the isobaric tags for relative and absolute quantitation technique. Fertil. Steril. 100, 505-510. doi: 10.1016/j.fertnstert.2013.04.008

Yan, J. X., Devenish, A. T., Wait, R., Stone, T., Lewis, S., and Fowler, S. (2002). Fluorescence two-dimensional difference gel electrophoresis and mass spectrometry based proteomic analysis of Escherichia coli. Proteomics 2, 1682-1698. doi: 10.1002/1615-9861(200212)2:12<1682::AID-PROT1682>3.0.CO;2-Y

Yun, S. H., Choi, C. W., Kwon, S. O., Park, G. W., Cho, K., Kwon, K. H., et al. (2011). Quantitative proteomic analysis of cell wall and plasma membrane fractions from multidrug-resistant Acinetobacter baumannii. J. Proteome Res. 10, 459-469. doi: $10.1021 /$ pr101012s

Zhang, J., Song, M. Q., Zhu, J. S., Zhou, Z., Xu, Z. P., Chen, W. X., et al. (2011). Identification of differentially-expressed proteins between early submucosal non-invasive and invasive colorectal cancer using 2D-DIGE and mass spectrometry. Int. J. Immunopathol. Pharmacol. 24, 849-859.

Zhu, W., Smith, J. W., and Huang, C. M. (2010). Mass spectrometry-based label-free quantitative proteomics. J. Biomed. Biotechnol. 2010, 840518. doi: $10.1155 / 2010 / 840518$

Conflict of Interest Statement: The authors declare that the research was conducted in the absence of any commercial or financial relationships that could be construed as a potential conflict of interest.

Received: 25 June 2014; accepted: 11 September 2014; published online: 26 September 2014.

Citation: Tiwari V and Tiwari M (2014) Quantitative proteomics to study carbapenem resistance in Acinetobacter baumannii. Front. Microbiol. 5:512. doi: 10.3389/fmicb.2014.00512

This article was submitted to Antimicrobials, Resistance and Chemotherapy, a section of the journal Frontiers in Microbiology.

Copyright $\odot 2014$ Tiwari and Tiwari. This is an open-access article distributed under the terms of the Creative Commons Attribution License (CC BY). The use, distribution or reproduction in other forums is permitted, provided the original author(s) or licensor are credited and that the original publication in this journal is cited, in accordance with accepted academic practice. No use, distribution or reproduction is permitted which does not comply with these terms. 